SULL'EFFETTO VOLTA.

Nota del Dott. Q. MAIORANA ').

1. Le due teorie per la spiegazione dell'effetto Volta. La esperienza fondamentale di Volta è ancora oggi interpretati in guise diverse dai fisici. Da un canto si hanno i sostenitori della Teoria del Contatto, che sono i più, e dall'altro i fautori di una teoria immaginata dal Prof. Lodge nel 1884, la teoria della tendenza chimica. $\dot{\mathrm{E}}$ dunque bene, se ci si vuol mantenere estranei a questa polemica, parlare semplicemente di effetto Volta, senza preoccuparci della sua origine intima.

L'effetto Volta puó essere assimilato ad una differenza di potenziale tra i vari metalli, e questa differenza non iscomparisce mettendoli in contatto. Si puó dunque parlare di potenziale naturale dei diversi metalli. Cosi $\mathrm{Zn}$ e Pt posseggono, rispettivamente, potenziali naturali che differiscono per 1 volt. Per lo studio di questi potenziali, occorre aver cura dello stato superficiale dei conduttori, ed occorre osservarli ponendo questi in contatto secco, giacchè debbono escludersi le azioni chimiche, molecolari, oltrechè le termiche e le meccaniche. Il potenziale naturale caratterizza dunque il corpo, nel suo stato di quiete, quando cioè queste azioni non arvengono, o non avverrebbero in seguito ad aver stabilito congiunzioni metalliche.

Pur won volendo entrare in discussione, sull' interpretazione dell'effetto Volta devesi riconoscere che, di fronte alla semplicità del principio su cui si basa la teoria del contatto, si trova una idea così complicata nei suoi particolari, che difficilmente può essere afferrata da chi si prova a leggere le memorie di Lodge, per la prima volta.

Le cariche che vengono osservate mediante la esperienza fondamentale di Volta, sarebbero dovute, secondo le vedute di

1) Risssunto fatto dall' A. delle Note puhblicste nei Rendiconti dei Lincei il 19 agosto, i] 2 il 16 Settembre 1900. 
Lodge, all' azione dell'ossigeno sui due metalli. Ma non è necessario, egli dice, che realmente avvenga alcuna azione chiInica, solo occorre qhe vi sia la possibilitá di ciò. E così resterebbe spiegato perchè l'effetto Volta si riscontri sempre nella stessa misura, anche nei vuoti più spinti, o in bagni li. quidi isolanti. In entrambi questi due casi, si troverebbe in presenza del metallo ossidabile, un numero di molecole sufficiente a conferire allo zinco il potenziale che si osserva. Ma tutto ciò è certamente artificioso, e l'artificio si spinge al punto di riconoscere come conclusione, esperienze che realmente non dicono nulla di nuovo. Il Sig. Spier's (Phil. Mag. Jan. 1900, p. 70) ha trovato che una coppia FePt, quando venga riscaldata vivamente in una atmosfera di $\mathrm{H}$, scende dal valore di +0.37 nell'aria a $-0,60$ volt. Secondo l'autore con cio si sarebbe posta la coppia in una atmosfera di solo idrogeno, e per conseguenza il valore della $\mathrm{f}$. e. $\mathrm{m}$. di contatto è diverso. Ma è noto (Kelvin, Nature, Apr. 1881) che il Pt nell'idrogeno, diventa più ppsitivo. L'esperienza dello Spiers puo dunque spiegarsi ammettendo un cambiamento dello stato superficiale dei due metalli. In un caso solo dovrebbesi riconoscere la giustezza delle vedute di Lodge, quando cioè si fosse dimostrato sperimentalmente, che due metalli diversi, a superficie ben terse e privi di qualsiasi atmosfera ambiente ed aderente, dieno luogo ad un effetto Volta nullo.

2. Nuovo metodo per la misura dell' effetto Volta. - Un filo di quarzo argentato $F$ (fig. 1), è sospeso da due sfere for.

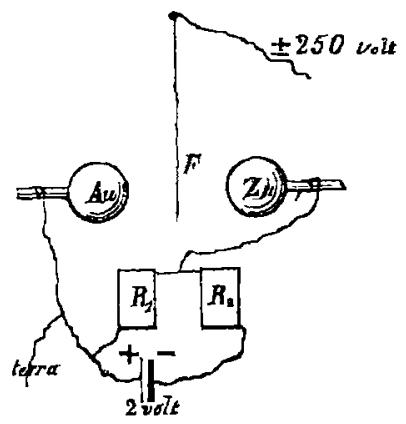

Fig. 1. 
mate dai due metalli su cui si sperimenta. Questo sistema è chiuso in una scatola di vetro con viti di livello, e dall'esterno di essa, si possono stabilire comunicazioni metalliche con ciascuno di quei tre conduttori. Due cassette di resisten $\% \mathbf{R}_{1}, \mathbf{R}_{\mathbf{q}}$ eguali e, ciascuna, da 100 ohm e con le suddivisioni sino ai decimi, sono disposte insieme con un accumulatore di grande capracità e di conosciuta f. e. m., come nel disegno.

Le spine di una delle cassette sono soppresse, talchè, se si ha l'arvertenza di servirsi di una medesima spina, per togliere sempre una resistenza di determinato valore, sia di $R_{1}$ che di $R_{2}$, la complessiva resistenza offerta dalle cassette, al passaggio della corrente, è sempre di $100 \mathrm{ohm}$. Ma a seconda della posizione delle spine, il potenziale della sfera di $\mathrm{Zn}$ puó variare da 0 sino a 2 volt circa, e ció gradatamente per cinquecentesimi volt. Il filo $\mathrm{F}$ puó essere portato ad un potenziale positivo o negativo di 250 volt circa, per mezzo di una batteria di 100 piccoli accumulatori. Sperimentalmente si procede cosi : Si elettrizaa $F$ in un senso quaisiasi, e si osserva da quale parte cade. Lo si elettrizza allora con elettricitá di senso contrario. Se cade dalla stessa parte, ció è doruto a dissimetria, che viene corretta con le riti di livello. Dopo pochi tentativi, si arriva a far sì che si puó far cadere $F$ sia a destra che a sinistra, a seconda del segno di elettrizzazione. Ció quando tulte le spine sono sulla cassetta $R_{1}$, ossia questa ̀̀ esclusa. Si comincia quindi a levare qualche spina da $R_{1}$ e porla in $R_{2}$, e, con qualche tentativo, si arriva cosi ad eguagliare $\mathrm{i}$ potenziali dei due metalli. F resta allora immobile o devia sempre dalla stessa parte, indipendentemente dal segno della carica. Se $v$ è il voltaggio dell'accumulatore, la differenza di potenziale tra $\mathrm{i}$ due metalli ̀̀

$$
e=v \frac{\mathbf{R}_{1}}{\mathrm{R}_{1}+\mathrm{R}_{2}}=\frac{1}{100} v \mathrm{R}_{1} .
$$

Più è grande il potenziale di elettrizzazione di F, maggiore è la sensibilità del metodo, ma é bene che esso non sia tale da produrre attrazioni troppo brusche. Analizzare le leggi che governano queste attrazioni, sarebbe forse cosa impossibile se fatta rigorosamente, ma un caso teorico semplicissimo, mo- 
stra come vi sieno interessanti considerazioni da fare su di un poblema di tal genere. Su di una retta, si immaginino tre masse elettriche isolate $+a,+q,-a$, portate da sfere piccolissime. Le due sfere $a$ sieno egualmente distanti per il seginento $r$, dalla sfera $q$. Questa è portata da un filo sottile, molto lungo e verticale. Se essa si sposta dalla posizione centrale per le azioni delle due sfere $a$, tende a ritornarvi con una forza che è proporzionale allo spostamento. E facile dimostrare che l'equilibrio di $q$ non è possibile, in una guisa stabile, che nel solo caso in cui essa non si sposti dalla posizione centrale per un segmento, minore di una certa frazione massima di $r$, sia a destra che a sinistra. Questa frazione è

$$
\pm 0,39332 r \text {. }
$$

Il caso pratico è molto più complicato, perchè le due sfere non sono abbastanza piccole, e perchè fenomeni di induzione, dovuti alla presenza del filo di quarzo elettrizzato richiamano sulle sfere, quantità di elettricitá superiori alle preesistenti. Entrambe queste cause contribuiscono a deprimere il valore delle due deviazioni critiche, ma che anche qui esse si abbiano, è facile verificare sperimentalmente. Così nel caso di due sfere di $25 \mathrm{~mm}$. di diametro, i cui centri distano $6 \mathrm{~cm}$., le due deviazioni critiche sono solo di circa $2 \mathrm{~mm}$. a destra e a sinistra, della posizione centrale di riposo.

3. Influenza dello stato superficiale sull'effetto Volta. Il metodo suaccennato puó essere in certi casi adoprato con vantaggrio. Sia quando la superficie dei conduttori è piccola, o sia che, per necessità sperimentali le dimensioni dell' apparecchio debbano essere piccole, esso si presta a preferenza di qualsiasi altro. Si puó cosi studiare l'influenza dello stato superficiale sull'effetto Volta.

Una coppia di dischi metallici di $25 \mathrm{~mm}$. di diametro di Zn e di ottone dorato, è sostituita alle sfere della fig. I. Se i due dischi sono entrambi tenuti ad una notevole distanza dal filo di quarzo, è evidente che le azioni di tutti gli elementi delle loro superficie affacciate verso il filo, sul filo stesso, sono poco differenti ed il potenziale di compensazione, indicato dalle cassette di resistenza, è sensibilmente la media differenza di 
potenziale, tra i varii punti dei due dischi. Ma se essi vengono avvicinati di molto al filo, su questo agiscono principalmente gli elementi di superficie più vicini. Si abbassi ora il filo di quarzo, in guisa che la sua estremità inferiore sia all' altezza dei bordi inferiori dei due dischi; la differenza di potenziale misurata è allora

$$
e=0,91 \text { volt. }
$$

Innalzando il filo, con la estremità ad $\%$ di diametro dal bordo inferiore, si trova

$$
e=0,82 \text {. }
$$

Innalzando ancora, sino a porla quasi fuoridei due dischi,

$$
e=0,97 \text {. }
$$

Nella prima esperienza agiscono sul filo tanto le parti centrali, che i bordi inferiori e superiori dei due dischi. Nella seconda le parti centrali, e i soli bordi superiori, e nella terza i soli bordi superiori. Si vede dunque che la differenza di potenziale dei due dischi, è minore al centro e maggiore alla periferia. Questo risultato, che tiene ai dischi adoperati, è peró abbastanza generale; giacchè è difficile pulire egualmente, in tutti i suoi punti, una superficie circolare e piana.

Così similmente, una serie di coppie di sfere eguali con diametri crescenti da $18 \mathrm{~mm}$. sino a $55 \mathrm{~mm}$., presenta differenze di potenziale che crescono abbastanza regolarmente da 0,50 a 0,80 volt. E ció per quanta cura abbia messo il meccanico nel costruirle. Solo, pulendo ripetutamente, e con maggior velocità di rotazione, le sfere di $18 \mathrm{~mm}$., si può portarle alla differenza di potenziale di $0,8-0,9$ volt.

4. Infuenza delle basse temperature sull Effetto Volta. - Tanto che si ammetta la teoria di Volta, riconosciuta tuttora da Kelvin, secondo la quale il potenziale naturale dei metalli è dovuto ad una proprietà molecolare di ciascun corpo, indipendentemente dal mezzo in cui esso è immerso, o altra teoria, simile a quella di Lodge, secondo cui l'effetto Volta si deve all'affinità chimica del mezzo per i metalli, è prevedibile che, coll'abbassarsi della temperatura, l'effetto Volta venga depresso. Ma è cosa difficile studiare ció, con l'esperienza, giac- 
chè, come fa notare Pellat, è difficile mutare la temperatura di un conduttore senza mutarne lo stato superficiale. Questo inconveniente, che principalmente si fa sentire quando si scaldano metalli così facilmente ossidabili come lo zinco, non è più a deplorarsi, o almeno è molto meno da temersi raffreddando i metalli stessi.

Un'ampolla di vetro della forma indicata nella fig. 2, porta il filo di quarzo $F$ e i due dischi dei metalli che si studiano.

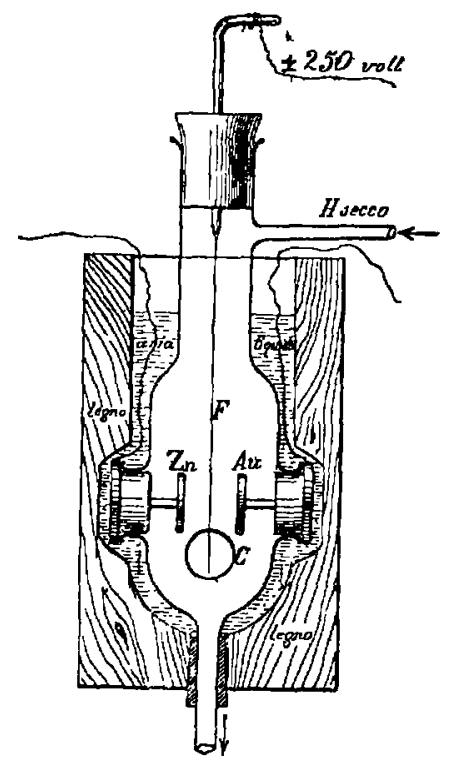

Fig. 2.

Un astuccio di legno circonda l'apparecchio, ed in esso si puó versare dell'aria liquida. La coppia bimetallica viene cosi portata alla temperatura di circa $-180^{\circ} \mathrm{c}$. Afflnchè un raffreddamento così energico, non generi la liquefazione dell'atmosfera contenuta nell'ampolla, si fa circolare in questa continuante dell'idrogeno puro e secco. Ecco i principali risultati ottenuti:

$\begin{array}{lccc} & \text { Zn Au } & \text { Al Au } & \text { Fe Au } \\ \text { Nell'atmosfera } & 0,88 & 1,05 & 0,41 \\ \text { Nell'H secco, dopo 24 ore } & 0,80 & 0,93 & 0,37 \\ \text { Versata l'aria liquida } & 0,05 & 0,09 & \text { non misurabile } \\ \text { Dopo un'ora } & 0,73 & 0,82 & 0,25 .\end{array}$


Si vede dunque che la differenza di potenziale delle varie coppie decresce enormemente con la temperatura; risale in seguito, ma quando i metalli riprendono la temperatura ambiente essa è alquanto minore di prima. Ciò è dovuto a leggera alterazione dello stato superficiale, ed in ogni modo questo fenomeno secondario, è piccolo di fronte a quello che si studia. Si può dunque prevedere che l'effetto Volta tende ad annullarsi coll'abbassarsi della temperatura, e che probabilmente a $-273^{\circ}$ c. esso non ha piú luogo.

I risultati ottenuti per le coppie di metalli citate, sono cosi concordi da lasciar credere che verisimilmente qualsiasi altra coppia si comporti alla stessa guisa. Se ciò è vero, costruendo le curve dei potenziali dei diversi metalli, con l'abbassarsi della temperatura, esse non si incontrano mai, ma finiscono tutte per toccare a $-273^{\circ} \mathrm{c}$. un certo asse delle $x$ che rappresenta il potenziale posseduto dai corpi a quella temperatura. E ciò permette anche di concludere che: la serie di Volta resta immutata coll' abbassarsi della temperatura. Questi abbassamenti non hanno altro effetto, che quello di deprimere il valore delle differenze di potenziale di ciascuna coppia.

Perchè si abbia la conoscenza reale delle curve predette, occorrerebhe conoscere il valore del potenziale dei corpi allo zero assoluto; valore che verrebbe assunto come zero della scala dei potenziali. Ma la ricerca di questo valore è a mio avviso alquanto difficile. Occorrerebbe infatti paragonare un campione di ciascun metallo a temperatura ambiente, con altri campioni di egual natura, ma tenuti a temperature successivamente decrescenti. Se questa ricerca tosse possibibile, si arriverebbe a scoprire se tra la serie naturale dei metalli ve ne sia qualcuno, che presenti variazioni di potenziale nulle o quasi; il suo potenziale sarebbe il cercato. Di tutti gli altri metalli, quelli che hanno potenziale superiore a questo, diventerebbero meno positive con l'abbassarsi della temperatura, gli altri meno negativi.

Considerazioni che faccio nel testo originale di questo lavoro, mi funno però credere che probabilmente tulti $i$ metalli diventano piu positivi, coll'innalzarsi della temperatura. 
5. Attrazione fra metalli eterogenei. - Feci giả vedere che a causa della differenza di potenziale tra due metalli eterogenei, essi debbón attirarsi, e indicai alcune disposizioni sperimentali atte a dimostrare questo fatto. Ricorrendo all'uso delle frange di interferenza, ho potuto ora misurare il fenomeno in maniera molto più precisa. Una piccola bilancia, sensibile al centomillesimo di milligrammo, ha servito a questo scopo. Essa deve essere adattata per mezzo dell' imboccatura $\mathrm{T}$ (fig. 3), alla parte ottica del dilatometro Abbe-Pulfrich co-

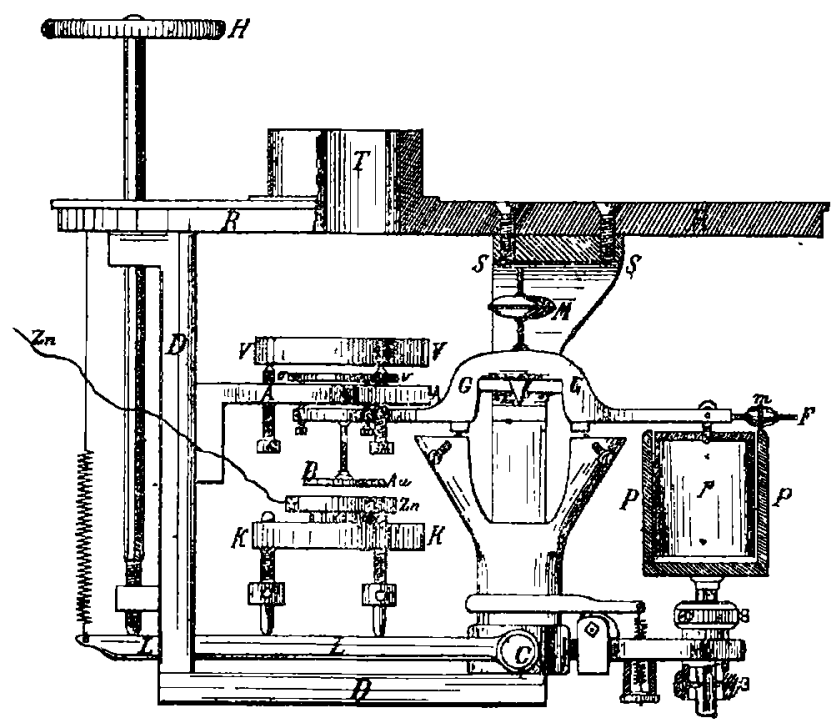

Fig. 3.

struito dalla casa Zeiss di Jena '), che, come è noto, utilizza il principio di Fizeau per lo studio della dilatazione dei solidi. Una piccolì staffa $S$ è chiusa, nella sua parte inferiore, da un piano orizzontale di acciajo temperato, che serve di appoggio al coltello pure di acciajo del giogo (7. Un disco circolare A, fissato all' armatura D, porta alla sua periferia tre viti verti-

(1) Per intendere la descrizio:e di queste osperionze occorre ben conoscere il predetto dilatometro. Yedi: Zutsch. fur Istrumentenkundo: Ueber das Abbo-Fizeau'sche Dilatometer v. Dr C. Pulfrich, 1893 $\vee$ 13. p. 364. La bilancia è stata costruita, su miei disegni, dal Sig. E. Squarzent1, meccanico dell Istituto Fisico di Ruma. 
cali, su cui appoggia un piano di vetro $\mathrm{V}$ a facce piane e non parallele. Il disco A è forato in guisa da lasciar passare altro tre viti portate dal giogo $G$, e sulle quali appoggia una seconda lastrina $v$, la cui faccia inferiore è smerigliata. Al giogo è attaccato il disco B di ottone dorato. Sotto a questo si trova il disco di zinco.

Colla vite $\mathrm{H}$ si può cambiare la distanza tra $\mathrm{Au}$ e $\mathrm{Zn}$. Il braccio di destra porta un raso $p$, che scorrendo senza toccarlo dentro un secondo vaso $\mathrm{P}$, smorza rapidamente le oscillazioni della bilancia. Due masse $\mathrm{M}$, $m$, mobili, su viti, servono a cambiare rispettivamente, la sensibilità e la posizione di riposo della bilancia. Il congegno per l'arresto e pel sollevamento del giogo, indicato in figura, si intende senz'altro. La luce proveniente da una sorgente monocrornatica, viene a cadere sulle due lastrine $\mathrm{V}, v$, convenientemente regolate $e$, da esse rinviata, arriva all'occhio dello sperimentatore, dando luogo alla formazione delle frange. Si pone lo $\mathrm{Zn}$ in comunicazione con la massa della bilancia. Con ció la differenza di potenziale tra $\mathrm{Zn}$ e Au è quella dei loro potenziali naturali. Si comincia allora ad avvicinare, mediante le vite $\mathrm{H}$, lo $\mathrm{Zn}$ all'Au, e ció molto lentamente. Si arriva cosi a portare i due dischi a circa $0,5 \mathrm{~mm}$. di distanza senza che si tocchino, $\mathrm{e}$ con ciò 12 o 15 frange (stria verde del mercurio) escono dal campo a causa dell' attrazione dei due dischi. Ciò corrisponde ad uno spostamento del giogo di circa 6 micron, $\theta$ ad una sensibilita della bilancia di $1 \mathrm{~mm}$. di spostamento per $1 / 10 \mathrm{di} \mathrm{mg}$.

Lo spostamento delle frange può essere in parte dovuto alla attrazione newtoniana dei due dischi, ( 1 o 2 frange, se i dischi pesano $10 \mathrm{gr}$. ciascuno). Ma è facile evitare ció, co. struendo i dischi molto sottili. Siano A, B (fig. 4) i due dischi di oro e zinco dell'apparecchio, la cui differenza di potenziale sia $v$. In conseguenza del lavoro di attrazione, il disco A viene in C. Mediante una sorgente elettrica di forza elettromotrice $V$, si porti il disco A al potenziale $\mathrm{V}+v$; esso si abbassa ancora venendo in $\mathrm{D}$. Scambiando $\mathrm{i}$ poli della sorgente, il disco d'oro acquista (in valore assoluto) il potenziale $r-v$, e risale in F. In tutto ció si suppone che gli spostamenti del disco A, sieno piccoli di fronte alla distanza A B, talchè la distanza $d$ 
dei due dischi possa ritenersi immutata. Diciamo $x, a, b$ i tre spostamenti A C, CD, C E. Di essi, gli ultimi due possono mi-

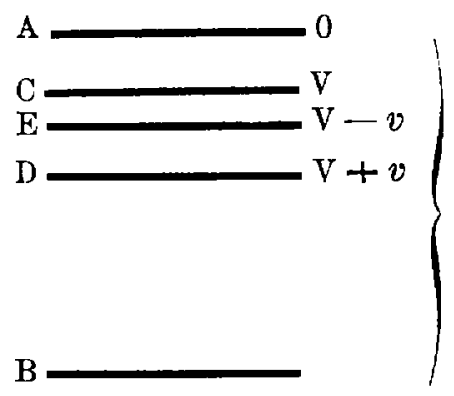

Fig. 4.

surarsi col mezzo delle frange, ed il primo è incognito. La forza attrattiva elettrica quando il disco d'oro è in $\mathrm{C}$ è :

$$
\mathrm{F}=h x=\frac{\mathrm{R}^{2}}{8 d^{2}} v^{2}
$$

dove $h$ è una costante inerente alle condizioni di sensibilità della bilancia, sicchè :

$$
x=\frac{l}{h} \frac{\mathrm{R}^{2}}{8 d^{2}} v^{2} .
$$

Similmente :

$$
a+x=\frac{\mathrm{R}^{2}}{8 d^{2} h}(\mathrm{~V}+v) ; \quad b+x=\frac{\mathrm{R}^{2}}{8 d^{2} h}(\mathrm{~V}-v)^{2} .
$$

Diciamo:

$$
r=\frac{\mathrm{R}^{2}}{8 d^{2} h}
$$

Si avrd

$$
x=r v^{2}, \quad a+x=r(\mathrm{~V}+v)^{2}, \quad b+x=r(\mathrm{~V}-v)^{2} .
$$

In questo sistema di equazioni, vi sono tre incognite; $x, r, v$. Risolvendo rispetto a $v$ si ha:

$$
v=\frac{\mathrm{V}}{2} \frac{a-b}{a+b}
$$

serie IV. Vol. XII. 
Se dunque si osservano gli spostamenti $a, b$ prodotti da un potenziale ausiliario qualsiasi $\mathrm{V}$, mediante la formula precedente, si puó conoscere la differenza di potenziale dei due metalli. Ma alcuni casi particolari offrono metodi di misura molto semplici.

Suppongasi $a=-3 b$, allora $v=-V$. Se dunque, mediante una nota disposizione, si rende variabile il potenziale ausiliario e conosciuto $\mathrm{V}$, quando esso diviene eguale e di segno contrario alla incognita $v$, il numero delle frange che escono caricando l'oro con elettricità di un certo segrno, è triplo di quello delle frange che rientrano, elettrizzandolo con segno opposto. Sicchè per tentativi si trova direttamente il valore di $v$.

Lo stesso risultato si ha se $b=-3 a$.

Per $a=0, v=-\frac{\mathrm{V}}{2}$. Se dunque il potenziale compensante è doppio e di segno contrario del cercato, non si ha nessuno spostamento nelle fi'ange. Questo è il metodo più sicuro e più esatto di misura. Esso è infatti metodo di riduzione a zero, e non si ha spostamento nella posizione relativa dei due dischi, per cui la quantità $a$ rimane costante, e non v'è da temere alcun errore dovuto alla forza newtoniana.

Le stesse considerazioni valgono se $b=0$.

Mediante la conoscenza degli spostamenti $a, b$ sotto l'azione di un potenziale ausiliario $\mathrm{V}$, si può anche determinare il valore di $\boldsymbol{x}, \boldsymbol{o}$, in altri termini, la forza incognita che agisce tra i due dischi. Dal sistema di equazioni superiore si ha infatti :

$$
\mathrm{F}=\frac{h}{8} \frac{(a-b)^{2}}{a+b} \text {. }
$$

E più semplicemente, nel caso in cui il potenziale compensante è doppio della differenza di potenziale naturale,

$$
\mathrm{F}=\frac{h b}{8} \text {. }
$$

Ed infine, risolvendo rispetto ad $r$, si ha:

$$
r=\frac{a+b}{2 \mathrm{~V}^{2}} \text {. }
$$


Ciò̀ :

$$
d^{2}=\frac{\mathrm{R}^{2} \mathrm{~V}^{2}}{4 h(a+b)}
$$

Ossia, conosciuta la sensibilitì $h$ della bilancia, si puó mediante la precedente relazione determinare, in ogni esperienza, la distanza $d$ dei due dischi.

\section{LIBRI NUOVI}

\section{LEÇONS DE GRINIE PYSIOUR,}

di I. H. VAN'T HOFF.

(Traduzione francese di M. Corvisy, Parigi, A. Hermann, 1900).

Col terzo volume ora pubblicato si compie l'importante opera del Van't Hoff sulla fisico-chimica (cfr. N. Cim. (4) 8, p. 318 , e 10 p. 451 .

Dopo aver trattato, nei primi due volumi, della dinamica e della statica chimica, in questo sono esposte le relazioni fra le proprietd e la composizione dei corpi.

Il libro comincia con l'esposizione delle relazioni fra le proprietá fisiche e la composizione, e tratta delle relazioni di volume - sia collegative che additive e costitutive - di pressione, di temperatura, e infine le relazioni calorimetriche, ottiche e di capillarità.

Vongono poi studiate le relazioni fra la composizione e le proprieta chimiche, e il v. Hoff fa osservare che in questo campo è più difflcile che nel precedente di ridurre le proprietá studiate ad un'espressione numerica indipendente dalle condizioni nelle quali ci si pone. Per es. non si sa esprimere con una legge generale l'influenza che la temperatura esercita sulla velocità delle reazioni.

Il van't Hoff in questa parte del libro mostra come l'insieme delle relazioni fra le proprieta chimiche e la composizione possa esprimersi mediante le seguenti proposizioni genel'ali : 